\title{
A FUSS-TYPE FAMILY OF POSITIVE DEFINITE SEQUENCES
}

\author{
WOJCIECH MŁOTKOWSKI AND KAROL A. PENSON
}

\begin{abstract}
We study a two-parameter family $a_{n}(p, t)$ of deformations of the Fuss numbers. We show a sufficient condition for positive definiteness of $a_{n}(p, t)$ and prove that some of the corresponding probability measures are infinitely divisible with respect to the additive free convolution.
\end{abstract}

\section{INTRODUCTION}

The aim of the paper is to study a two-parameter family of sequences $a_{n}(p, t), p, t \in \mathbb{R}$, defined by (17), which can be regarded as deformation of the Fuss numbers. Assuming that $p \geq 0$ we prove that the sequence $a_{n}(p, t)$ is positive definite if and only if $p \geq 1$ and $g(p) \leq t \leq 2 p /(p+1)$, where $g(p)$ is defined by 25$)$. We conjecture that the assumption that $p \geq 0$ is redundant.

The case $t=2 p /(p+1)$ is particularly interesting by connections with the work [6] of M. Bousquet-Mélou and G. Schaeffer. They introduced the notion of constellation as a tool for studying factorization problems in the symmetric groups. For $p \geq 2$ a $p$-constellation is a 2-cell decomposition of the oriented sphere into vertices, edges and faces, with faces colored black and white in such a way that:

- all faces adjacent to a given white face are black and vice versa,

- the degree of any black face is $p$,

- the degree of any white face is a multiple of $p$.

A constellation is called rooted if one of the edges is distinguished.

The number of rooted $p$-constellations formed of $n$ polygons, counted up to isomorphism, is given by

$$
C_{p}(n):=\left(\begin{array}{c}
n p \\
n
\end{array}\right) \frac{(p+1) p^{n-1}}{(n p-n+1)(n p-n+2)},
$$

$p \geq 2, n \geq 1$, see Corollary 2.4 in [6]. Some of these sequences appear in the On-line Encyclopedia of Integer Sequences (OEIS) [26], namely: $C_{2}=A 000257, C_{3}=A 069726$, $C_{4}=A 090374$.

We will prove that the probability distribution $\eta(p, t)$ corresponding to positive definite sequence $a_{n}(p, t)$ is absolutely continuous, except for $\eta(1,1)=\delta_{1}$, and the support of $\eta(p, t)$ is $\left[0, p^{p}(p-1)^{1-p}\right]$. The density function will be denoted $f_{p, t}(x)$. For $p=2$ and $p=3$ we compute the $R$-transform of $\eta(p, t)$. We prove that $\eta(2, p)$ (resp. $\eta(3, t)$ ) is infinitely divisible with respect to the additive free convolution if and only if $1 \leq t \leq 4 / 3$ (resp. $1 / 2 \leq t \leq 3 / 2$ ).

Finally, let us record some other sequences from OEIS which are related to this work: $A 005807: 2 a_{n}(2,1 / 2)$ (sums of adjacent Catalan numbers), $A 007226: 2 a_{n}(3,1 / 2)$

2010 Mathematics Subject Classification. Primary 44A60; Secondary 33C20, 46L54.

Key words and phrases. Mellin convolution, Meijer $G$-function, free probability.

W. M. is supported by the Polish National Science Center grant No. 2012/05/B/ST1/00626. 
(studied in [15]), A007054: $3 a_{n}(2,4 / 3)$ (super ballot numbers), $A 038629: 3 a_{n}(2,2 / 3)$, A000139: $2 a_{n}(3,3 / 2), A 197271: 5 a_{n}(4,8 / 5), A 197272: 3 a_{n}(5,5 / 3)$. In Section 4 we also encounter sequences $A 022558$ and $A 220910$.

\section{Fuss Numbers}

The Fuss-Catalan numbers $\left(\begin{array}{c}n p+1 \\ n\end{array}\right) \frac{1}{n p+1}$ have several combinatorial applications, see [9, 17, 2, 27, 6, 24]. They count for example:

(1) the number of ways of subdividing a convex polygon, with $n(p-1)+2$ vertices, into $n$ disjoint $p+1$-gons by means of nonintersecting diagonals,

(2) the number of sequences $\left(a_{1}, a_{2}, \ldots, a_{n p}\right)$, where $a_{i} \in\{1,1-p\}$, with all partial sums $a_{1}+\ldots+a_{k}$ nonnegative and with $a_{1}+\ldots+a_{n p}=0$,

(3) the number of noncrossing partitions $\pi$ of $\{1,2, \ldots, n(p-1)\}$, such that $p-1$ divides the cardinality of every block of $\pi$,

(4) the number of $p$-cacti formed of $n$ polygons, see [6].

The generating function:

$$
\mathcal{B}_{p}(z):=\sum_{n=0}^{\infty}\left(\begin{array}{c}
n p+1 \\
n
\end{array}\right) \frac{z^{n}}{n p+1}
$$

satisfies

$$
\mathcal{B}_{p}(z)=1+z \mathcal{B}_{p}(z)^{p} .
$$

Recall also the Lambert's formula for the Taylor expansion of the powers of $\mathcal{B}_{p}(z)$ :

$$
\mathcal{B}_{p}(z)^{r}=\sum_{n=0}^{\infty}\left(\begin{array}{c}
n p+r \\
n
\end{array}\right) \frac{r z^{n}}{n p+r} .
$$

These formulas remain true for $p, r \in \mathbb{R}$ and the coefficients $\left(\begin{array}{c}n p+r \\ n\end{array}\right) \frac{r}{n p+r}$ (understood to be 1 for $n=0$ and $\frac{r}{n !} \prod_{i=1}^{n-1}(n p+r-i)$ for $\left.n \geq 1\right)$ are called two-parameter Fuss numbers or Raney numbers, see [9, 13, 22, 12, 8].

In some cases the function $\mathcal{B}_{p}$ can be written explicitly, for example

$$
\begin{aligned}
\mathcal{B}_{2}(z) & =\frac{2}{1+\sqrt{1-4 z}}=\frac{1-\sqrt{1-4 z}}{2 z}, \\
\mathcal{B}_{3}(z) & =\frac{3}{3-4 \sin ^{2} \alpha}, \\
\mathcal{B}_{3 / 2}(z) & =\frac{3}{(\sqrt{3} \cos \beta-\sin \beta)^{2}},
\end{aligned}
$$

where $\alpha=\frac{1}{3} \arcsin (\sqrt{27 z / 4}), \beta=\frac{1}{3} \arcsin (3 z \sqrt{3} / 2)$, see [16].

Fuss numbers also have applications in free probability and in the theory of random matrices, as moments of the multiplicative free powers of the Marchenko-Pastur distribution [1, 3, 13, 17, 18]. This implies that for $p \geq 1$ the sequence $\left(\begin{array}{c}n p+1 \\ n\end{array}\right) \frac{1}{n p+1}$ is positive definite. More generally, the sequence $\left(\begin{array}{c}n p+r \\ n\end{array}\right) \frac{r}{n p+r}$ is positive definite if and only if either $p \geq 0,0 \leq r \leq p$, or $p \leq 0, p-1 \leq r \leq 0$ or $r=0$, see [13, 16, 12, 8]. The case $r=0$ is trivial, as it gives the sequence $1,0,0,0, \ldots$, moments of $\delta_{0}$. The distributions 
corresponding to the second case, $p \leq 0, p-1 \leq r \leq 0$, are just reflections of those corresponding to $p \geq 0,0 \leq r \leq p$. It is a consequence of the identity

$$
\left(\begin{array}{c}
n p+r \\
n
\end{array}\right) \frac{r(-1)^{n}}{n p+r}=\left(\begin{array}{c}
n(1-p)-r \\
n
\end{array}\right) \frac{-r}{n(1-p)-r} .
$$

For $p>1, r>0$ we have the following integral representation:

$$
\left(\begin{array}{c}
n p+r \\
n
\end{array}\right) \frac{r}{n p+r}=\int_{0}^{c(p)} x^{n} W_{p, r}(x) d x,
$$

where where $c(p):=p^{p}(p-1)^{1-p}$, and $W_{p, r}$ can be described as:

$$
W_{p, r}(x)=\frac{(\sin (p-1) \phi)^{p-r-1} \sin \phi \sin r \phi}{\pi(\sin p \phi)^{p-r}},
$$

where

$$
x=\rho(\phi)=\frac{(\sin p \phi)^{p}}{\sin \phi(\sin (p-1) \phi)^{p-1}}, \quad 0<\phi<\pi / p .
$$

This function is nonnegative if and only if $r \leq p$, see [10, 18, 8].

If $p=k / l$ is a rational number, $1 \leq l<k$, then $W_{p, r}$ can be expressed in terms of the Meijer $G$-function (see [22, 14]):

$$
W_{p, r}(x)=\frac{r p^{r}}{x(p-1)^{r+1 / 2} \sqrt{2 k \pi}} G_{k, k}^{k, 0}\left(\frac{x^{l}}{c(p)^{l}} \mid \begin{array}{c}
\alpha_{1}, \ldots, \alpha_{k} \\
\beta_{1}, \ldots, \beta_{k}
\end{array}\right),
$$

$x \in(0, c(p))$ and the parameters $\alpha_{j}, \beta_{j}$ are given by:

$$
\begin{aligned}
& \alpha_{j}= \begin{cases}\frac{j}{l} & \text { if } 1 \leq j \leq l, \\
\frac{r+j-l}{k-l} & \text { if } l+1 \leq j \leq k,\end{cases} \\
& \beta_{j}=\frac{r+j-1}{k}, \quad 1 \leq j \leq k .
\end{aligned}
$$

Examples: Let us record formulas for the functions $W_{p, r}$ for $p=2,3,3 / 2$ and $r=1,2$. In these cases $W_{p, r}$ can be expressed as an elementary function, see [21, 22, 14].

$$
\begin{aligned}
& W_{2,1}(x)=\frac{1}{2 \pi} \sqrt{\frac{4-x}{x}}, \\
& W_{2,2}(x)=\frac{1}{2 \pi} \sqrt{x(4-x)},
\end{aligned}
$$

where $x \in(0,4)$. $W_{2,1}$ is the density of the Marchenko-Pastur distribution and $W_{2,2}$ is the Wigner's semicircle law translated by 2 . 


$$
\begin{aligned}
& W_{3,1}(x)=\frac{3(1+\sqrt{1-4 x / 27})^{2 / 3}-(4 x)^{1 / 3}}{3^{1 / 2} \pi(4 x)^{2 / 3}(1+\sqrt{1-4 x / 27})^{1 / 3}}, \\
& W_{3,2}(x)=\frac{9(1+\sqrt{1-4 x / 27})^{4 / 3}-(4 x)^{2 / 3}}{2 \pi 3^{3 / 2}(4 x)^{1 / 3}(1+\sqrt{1-4 x / 27})^{2 / 3}},
\end{aligned}
$$

where $x \in(0,27 / 4)$.

$$
\begin{aligned}
& W_{3 / 2,1}(x)=3^{1 / 2} \frac{\left(1+\sqrt{1-4 x^{2} / 27}\right)^{1 / 3}-\left(1-\sqrt{1-4 x^{2} / 27}\right)^{1 / 3}}{2(2 x)^{1 / 3} \pi} \\
& +3^{1 / 2}(2 x)^{1 / 3} \frac{\left(1+\sqrt{1-4 x^{2} / 27}\right)^{2 / 3}-\left(1-\sqrt{1-4 x^{2} / 27}\right)^{2 / 3}}{4 \pi}
\end{aligned}
$$

$$
\begin{gathered}
W_{3 / 2,2}(x)=\frac{3^{1 / 2}(2 x)^{5 / 3}}{8 \pi}\left(\left(1+\sqrt{1-4 x^{2} / 27}\right)^{1 / 3}-\left(1-\sqrt{1-4 x^{2} / 27}\right)^{1 / 3}\right) \\
+\frac{3^{1 / 2}(2 x)^{1 / 3}\left(x^{2}-1\right)}{4 \pi}\left(\left(1+\sqrt{1-4 x^{2} / 27}\right)^{2 / 3}-\left(1-\sqrt{1-4 x^{2} / 27}\right)^{2 / 3}\right),
\end{gathered}
$$

where $x \in(0,3 \sqrt{3} / 2)$. The function $W_{3 / 2,2}(x)$ is not nonnegative on its domain.

\section{A family of SEQUenCES}

For $p, t \in \mathbb{R}$ define sequence $a_{n}(p, t)$ as an affine combination of $\left(\begin{array}{c}n p+1 \\ n\end{array}\right) \frac{1}{n p+1}$ and $\left(\begin{array}{c}n p+2 \\ n\end{array}\right) \frac{2}{n p+2}$ :

$$
\begin{aligned}
a_{n}(p, t) & :=\left(\begin{array}{c}
n p+1 \\
n
\end{array}\right) \frac{t}{n p+1}+\left(\begin{array}{c}
n p+2 \\
n
\end{array}\right) \frac{2(1-t)}{n p+2} \\
& =\left(\begin{array}{c}
n p \\
n
\end{array}\right) \frac{n(2 p-t-p t)+2}{(n p-n+1)(n p-n+2)}
\end{aligned}
$$

in particular $a_{0}(p, t)=1$.

The generating function is

$$
t \mathcal{B}_{p}(z)+(1-t) \mathcal{B}_{p}(z)^{2}=\sum_{n=0}^{\infty} a_{n}(p, t) z^{n}
$$


For example:

$$
\begin{aligned}
t \mathcal{B}_{2}(z)+(1-t) \mathcal{B}_{2}(z)^{2} & =\frac{1-t+3 t z-2 z-(1-t+t z) \sqrt{1-4 z}}{2 z^{2}} \\
t \mathcal{B}_{3}(z)+(1-t) \mathcal{B}_{3}(z)^{2} & =\frac{9-12 t \sin ^{2} \alpha}{\left(3-4 \sin ^{2} \alpha\right)^{2}} \\
t \mathcal{B}_{3 / 2}(z)+(1-t) \mathcal{B}_{3 / 2}(z)^{2} & =\frac{9-6 t \sin ^{2} \beta+6 t \sqrt{3} \sin \beta \cos \beta}{(\sqrt{3} \cos \beta-\sin \beta)^{4}}
\end{aligned}
$$

where $\alpha=\frac{1}{3} \arcsin (\sqrt{27 z / 4}), \beta=\frac{1}{3} \arcsin (3 z \sqrt{3} / 2)$.

We are going to study positive definiteness of $a_{n}(p, t)$. First we observe

Proposition 3.1. If the sequence $a_{n}(p, t)$ is positive definite then

$$
2 p-p t-t^{2}+3 t-3 \geq 0 .
$$

In particular $t \neq 2$ and either $p \leq-3$ or $p \geq 1$.

Proof. The left hand side is just $a_{2}(p, t)-a_{1}(p, t)^{2}$.

\section{Examples.}

1. For $p=1$ we have $a_{n}(1, t)=1+n-n t$. Since $a_{2}(1, t)-a_{1}(1, t)^{2}=-(t-1)^{2}$, the sequence $a_{n}(1, t)$ is positive definite if and only if $t=1$. Note that $a_{n}(1,1)=1$ is the moment sequence of the one-point measure $\delta_{1}$.

2. For $t=2 /(p+1)$ we get

$$
a_{n}(p, 2 /(p+1))=\left(\begin{array}{c}
n p \\
n
\end{array}\right) \frac{2}{n p-n+2} .
$$

If $p>1$ then this is product of two positive definite sequences: $\left(\begin{array}{c}n p \\ n\end{array}\right)$ (see [16, 25]) and $2 /(n p-n+2)$.

3. Similarly, for $p>1, t=2 p /(p+1)$ the sequence

$$
a_{n}(p, 2 p /(p+1))=\left(\begin{array}{c}
n p \\
n
\end{array}\right) \frac{2}{(n p-n+1)(n p-n+2)} .
$$

is positive definite. Note that from (1) we have

$$
C_{p}(n)=\frac{(p+1) p^{n}}{2 p} a_{n}\left(p, \frac{2 p}{p+1}\right)
$$

so for $p \geq 1$ the sequence $C_{p}(n)$ is positive definite.

The sequence $a_{n}(p, t)$ is an affine combination of two sequences: $\left(\begin{array}{c}n p+1 \\ n\end{array}\right) \frac{1}{n p+1}$ and $\left(\begin{array}{c}n p+2 \\ n\end{array}\right) \frac{2}{n p+2}$. The former is positive definite for $p \geq 1$ and the latter for $p \geq 2$. This implies, that $a_{n}(p, t)$ is positive definite for $p \geq 2,0 \leq t \leq 1$. We are going to prove something stronger. Note that if $t_{1} \leq t_{2} \leq t_{3}$ and the sequences $a_{n}\left(p, t_{1}\right), a_{n}\left(p, t_{3}\right)$ are positive definite then so is $a_{n}\left(p, t_{2}\right)$ as their convex combination.

If we assume that $p>1$ then

where

$$
a_{n}(p, t)=\int_{0}^{c(p)} x^{n} f_{p, t}(x) d x,
$$

$$
f_{p, t}(x)=t W_{p, 1}(x)+(1-t) W_{p, 2}(x) .
$$


Figure 1. The density function $f_{3 / 2,1 / 5}(x)$

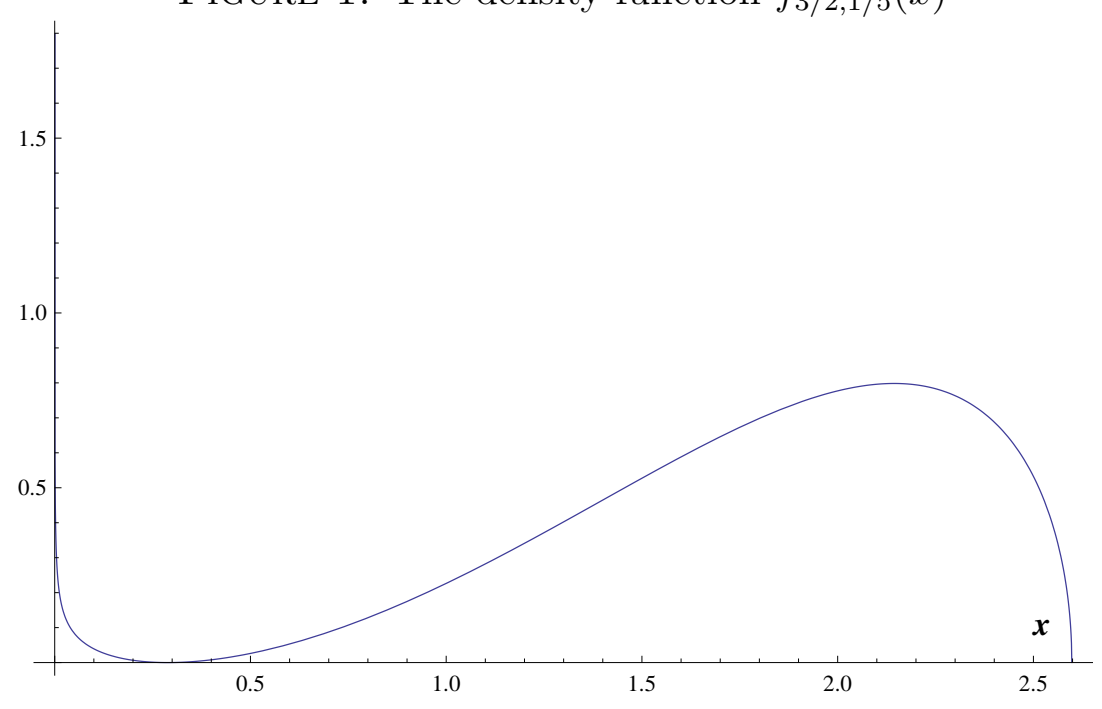

Then the positive definiteness of $a_{n}(p, t)$ is equivalent to the fact that $f_{p, t}$ is nonnegative on $(0, c(p))$. For example the function

$$
f_{2, t}(x)=\frac{t+x-t x}{2 \pi} \sqrt{\frac{4-x}{x}}
$$

is nonnegative on $(0,4)$ if and only if $0 \leq t \leq 4 / 3$.

By (6) we can write

$$
f_{p, t}(x)=\frac{\sin ^{2} \phi(\sin (p-1) \phi)^{p-3}[t \sin (p-1) \phi+2(1-t) \sin p \phi \cos \phi]}{\pi(\sin p \phi)^{p-1}}
$$

for $x$ as in (7). Define

$$
\begin{aligned}
\Psi_{p, t}(\phi) & =t \sin (1-1 / p) \phi+2(1-t) \sin \phi \cos \phi / p \\
& =(2-t) \sin \phi \cos \phi / p-t \cos \phi \sin \phi / p \\
& =(1-t) \sin (1+1 / p) \phi+\sin (1-1 / p) \phi
\end{aligned}
$$

Then the sequence $a_{n}(p, t)$ is positive definite if and only if $\Psi_{p, t}(\phi) \geq 0$ for $\phi \in[0, \pi]$. For $p \geq 1$ put

$$
g(p):=\min \left\{t \in \mathbb{R}: \Psi_{p, t}(\phi) \geq 0 \text { for all } 0<\phi<\pi\right\} .
$$

Since $\Psi_{p, t}(\pi)=t \sin (\pi / p)$ and $\Psi_{p, 1}(\phi)=\sin (1-1 / p) \phi$, we have $0 \leq g(p) \leq 1$ for all $p \geq 1$.

Proposition 3.2. The function $g$ is continuous on $[1, \infty), g(1)=1, g(p)=0$ for $p \geq 2$ and is strictly decreasing on $[1,2]$. In particular $g(3 / 2)=1 / 5$.

Proof. For $p=1$ we have $\Psi_{1, t}(\phi)=(1-t) \sin 2 \phi$, which implies $g(1)=1$. If $p \geq 2$ then $\Psi_{p, 0}(\phi)=2 \sin \phi \cos \phi / p$ is nonnegative for $\phi \in[0, \pi]$, which yields $g(p)=0$.

Now observe, that for fixed $t, \phi$, with $0 \leq t \leq 1,0<\phi \leq \pi$, the function $p \mapsto \Psi_{p, t}(\phi)$ is strictly increasing on $[1,2]$. Indeed, we can write

$$
\Psi_{p, t}(\phi)=2(1-t) \sin \phi \cos \phi / p+t \sin (\phi-\phi / p)
$$


and if $0<\phi \leq \pi$ then both the summands are increasing with $p \in[1,2]$. This implies, that $g(p)$ is strictly decreasing on $[1,2]$.

To prove continuity of $g$ assume that $1 \leq p_{1}<p_{2} \leq 2$ and put $t_{1}:=g\left(p_{1}\right), t_{2}:=g\left(p_{2}\right)$. Then $t_{1}>t_{2}, \Psi_{p_{1}, t_{1}}(\phi) \geq 0$ for all $\phi \in[0, \pi]$ and there is $\phi_{1}$, with $p_{1} \pi /\left(1+p_{1}\right)<\phi_{1}<\pi$, such that $\Psi_{p_{1}, t_{1}}\left(\phi_{1}\right)=0$. Then we have that $\Psi_{p_{2}, t_{1}}(\phi)>0$ for all $\phi \in(0, \pi]$. From the third expression in (24) we have that

$$
-c_{1}:=\sin \left(1+1 / p_{1}\right) \phi_{1}<0 .
$$

If we assume that $\left(p_{2}-p_{1}\right) \phi_{1}<c_{1} / 2$ then we have

$$
\left|\sin \left(1+1 / p_{1}\right) \phi_{1}-\sin \left(1+1 / p_{2}\right) \phi_{1}\right| \leq\left(1 / p_{1}-1 / p_{2}\right) \phi_{1}<c_{1} / 2
$$

and, consequently, $\sin \left(1+1 / p_{2}\right) \phi_{1}<-c_{1} / 2$.

If we take $t$, with $0 \leq t<t_{1}$, then

$$
\begin{gathered}
\Psi_{p_{2}, t}\left(\phi_{1}\right)=\Psi_{p_{2}, t}\left(\phi_{1}\right)-\Psi_{p_{1}, t_{1}}\left(\phi_{1}\right) \\
=\left(1-t_{1}\right)\left(\sin \left(1+1 / p_{2}\right) \phi_{1}-\sin \left(1+1 / p_{1}\right) \phi_{1}\right)+\left(\sin \left(1-1 / p_{2}\right) \phi_{1}-\sin \left(1-1 / p_{1}\right) \phi_{1}\right) \\
+\left(t_{1}-t\right) \sin \left(1+1 / p_{2}\right) \phi_{1} \leq\left(2-t_{1}\right)\left(p_{2}-p_{1}\right) \phi_{1}-\left(t_{1}-t\right) c_{1} / 2 .
\end{gathered}
$$

Hence, if

$$
\left(2-t_{1}\right)\left(p_{2}-p_{1}\right) \phi_{1}<\left(t_{1}-t\right) c_{1} / 2
$$

then $\Psi_{p_{2}, t}\left(\phi_{1}\right)<0$. This implies that

$$
g\left(p_{1}\right)-g\left(p_{2}\right)=t_{1}-t_{2} \leq 2\left(2-t_{1}\right)\left(p_{2}-p_{1}\right) \phi_{1} / c_{1} .
$$

and proves continuity of $g$.

For $p=3 / 2$ we can write

$$
\Psi_{3 / 2, t}(\phi)=\frac{\sin \phi / 3}{4}\left[(1-t)\left(5-8 \sin ^{2} \phi / 3\right)^{2}+5 t-1\right] .
$$

Note that $\sqrt{5 / 8}<\sqrt{3} / 2=\sin \pi / 3$, so, assuming that $0 \leq t \leq 1, \Psi_{3 / 2, t}$ attains its minimum on $[0, \pi]$ at $\phi=3 \arcsin \sqrt{5 / 8}$. This yields $g(3 / 2)=1 / 5$.

Now we are able to describe the domain of positive definiteness of the sequence $a_{n}(p, t)$, see Fig 2. The density function for the particular case $p=3 / 2, t=1 / 5$ is illustrated in Fig. 1.

Theorem 3.3. Suppose that $p \geq 0$. Then the sequence $a_{n}(p, t)$ is positive definite if and only if $p \geq 1$ and

$$
g(p) \leq t \leq \frac{2 p}{1+p}
$$

Proof. Fix $p \geq 1$. By the definition of $g(p)$ the sequence $a_{n}(p, t)$ is positive definite for $t=g(p)$ and not positive definite for $t<g(p)$.

We have already observed, that for $p \geq 1$ the sequence $a_{n}(p, 2 p /(p+1))$ is positive definite. If $t>2 p /(p+1)$ then $n(2 p-t-p t)+2<0$ and consequently $a_{n}(p, t)<0$ for all $n$ sufficiently large. Alternatively, we have $\Psi_{p, t}^{\prime}(0)=2 p-p t-t<0$ in this case, which implies $\Psi_{p, t}(x)<0$ for some $x \in(0, \pi / p)$. 
Figure 2. Domain of positive definiteness of the sequence $a_{n}(p, t)$

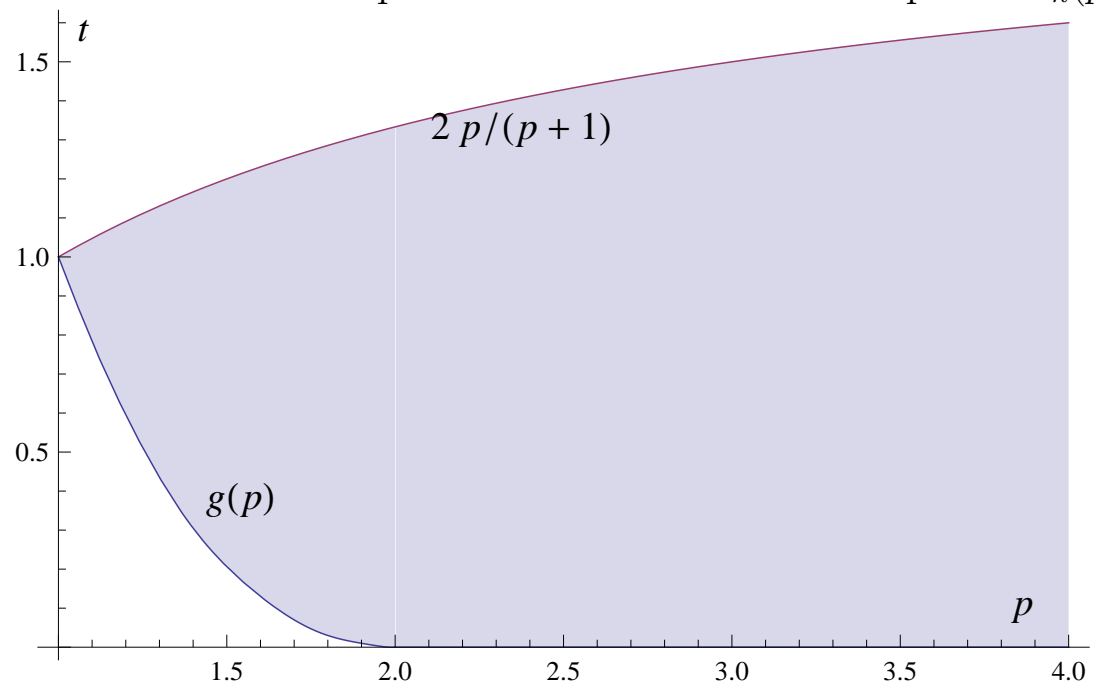

4. FreE TRANSFORMS

Throughout this section we assume that $p \geq 1$ and the sequence $a_{n}(p, t)$ is positive definite, i.e. $g(p) \leq t \leq 2 p /(p+1)$. Denote by $\eta(p, t)$ the corresponding distribution, i.e. $\eta(1,1)=\delta_{1}$ and $\eta(p, t)=f_{p, t}(x) d x$ on $\left[0, p^{p}(p-1)^{1-p}\right]$ for $p>1$. We are going to study relations of these measures with free probability.

Recall that for a compactly supported probability measure $\mu$ on $\mathbb{R}$, with the moment generating function

$$
M_{\mu}(z):=\sum_{n=0}^{\infty} z^{n} \int_{\mathbb{R}} x^{n} d \mu(x)=\int_{\mathbb{R}} \frac{1}{1-x z} d \mu(x),
$$

the $S$ - and $R$-transforms are defined by

$$
\begin{aligned}
& M_{\mu}\left(\frac{z}{1+z} S_{\mu}(z)\right)=1+z \\
& 1+R_{\mu}\left(z M_{\mu}(z)\right)=M_{\mu}(z) .
\end{aligned}
$$

Moreover, we have relation

$$
R_{\mu}\left(z S_{\mu}(z)\right)=z
$$

The coefficients $r_{n}(\mu)$ in the Taylor expansion $R_{\mu}(z)=\sum_{n=1}^{\infty} r_{n}(\mu) z^{n}$ are called free cumulants of $\mu$. It is known that $\mu$ is infinitely divisible with respect to the additive free convolution if and only if the sequence $\left\{r_{n+2}(\mu)\right\}_{n=0}^{\infty}$ is positive definite, see [28, 19].

For the distributions $\eta(p, t)$ we have

$$
M_{\eta(p, t)}(z):=\sum_{n=0}^{\infty} a_{n}(p, t) z^{n}=t \mathcal{B}_{p}(z)+(1-t) \mathcal{B}_{p}(z)^{2}
$$

Now we are going to compute the $S$-transform of $\eta(p, t)$. 
Proposition 4.1. For $p>1, g(p) \leq t \leq 2 p /(p+1)$ we have

$$
S_{\eta(p, t)}(w)=(2+2 w)^{1-p} \frac{\left(\sqrt{(2-t)^{2}+4(1-t) w}+t\right)^{p}}{\sqrt{(2-t)^{2}+4(1-t) w}+2-t} .
$$

Proof. From (3) we can derive relation

$$
\mathcal{B}_{p}\left(z(1+z)^{-p}\right)=1+z,
$$

see [13. Therefore

$$
M_{\eta(p, t)}\left(z(1+z)^{-p}\right)=t(1+z)+(1-t)(1+z)^{2} .
$$

If we substitute

$$
t(1+z)+(1-t)(1+z)^{2}=1+w
$$

then

$$
z=\frac{\sqrt{(2-t)^{2}+4(1-t) w}-2+t}{2(1-t)}=\frac{2 w}{\sqrt{(2-t)^{2}+4(1-t) w}+2-t}
$$

and

$$
1+z=\frac{\sqrt{(2-t)^{2}+4(1-t) w}-t}{2(1-t)}=\frac{2(1+w)}{\sqrt{(2-t)^{2}+4(1-t) w}+t},
$$

which combining with 28 yields $(31)$.

Now we are going to compute $R$-transform of $\eta(p, t)$ for $p=2$ and $p=3$. We will denote $r_{n}(p, t):=r_{n}(\eta(p, t))$.

4.1. The case $p=2$. The density function $f_{2, t}$ is given by $22,0 \leq t \leq 4 / 3$. From (31) we can compute the $R$-transform for $p=2$ :

Proposition 4.2. $R_{\eta(2,1)}=z /(1-z)$ and for $t \neq 1$

$$
R_{\eta(2, t)}(z)=\frac{1-t-2 z+3 t z-z^{2}+(t-1-z) \sqrt{1+z(2-4 t)+z^{2}}}{2(t-1)} .
$$

Moreover, $\eta(2, t)$ is infinitely divisible with respect to the additive free convolution if and only if either $t=0$ or $1 \leq t \leq 4 / 3$.

Proof. First we find $R_{\eta(2, t)}(z)$ by solving equation $S_{\eta(2, t)}\left(R_{\eta(2, t)}(z)\right) R_{\eta(2, t)}(z)=z$, equivalent with 30 , with the condition $R_{\eta(2, t)}(0)=0$. In particular $R_{2,0}=2 z+z^{2}$, which implies that $\eta(2,0)$ is infinitely divisible with respect to the additive free convolution.

Now we can find:

$$
\begin{aligned}
& r_{1}(2, t)=2-t \\
& r_{2}(2, t)=1+t-t^{2} \\
& r_{3}(2, t)=3 t^{2}-2 t^{3} \\
& r_{4}(2, t)=-4 t^{2}+10 t^{3}-5 t^{4} .
\end{aligned}
$$

Since

$$
r_{2}(2, t) r_{4}(2, t)-r_{3}(2, t)^{2}=t^{2}(t-1)(t-2)\left(t^{2}-2\right),
$$

for $0<t<1$ the distribution $\eta(2, t)$ is not infinitely divisible with respect to the additive free convolution. 
For $t \neq 1$ we have

$$
1+R_{\eta(2, t)}(z)=\frac{t-1-2 z+3 t z-z^{2}+(t-1-z) \sqrt{1+z(2-4 t)+z^{2}}}{2(t-1)}
$$

and $1+R_{\eta(2,1)}(z)=1 /(1-z)$. Then for $1<t \leq 3 / 2$ the function

$$
\frac{1+R_{\eta(2, t)}(1 / z)}{z}=\frac{(t-1) z^{2}-2 z+3 t z-1+(z(t-1)-1) \sqrt{1+z(2-4 t)+z^{2}}}{2(t-1) z^{3}}
$$

is the Cauchy transform of the probability distribution

$$
\frac{(1-t x+x) \sqrt{4 t(t-1)-(x-2 t+1)^{2}}}{2 \pi(t-1) x^{3}} d x,
$$

on the interval

$$
x \in\left[2 t-1-2 \sqrt{t^{2}-t}, 2 t-1+2 \sqrt{t^{2}-t}\right] .
$$

Therefore for $1<t \leq 4 / 3$

$$
r_{n}(2, t)=\int_{2 t-1-2 \sqrt{t^{2}-t}}^{2 t-1+2 \sqrt{t^{2}-t}} x^{n} \frac{(1-t x+x) \sqrt{4 t(t-1)-(x-2 t+1)^{2}}}{2 \pi(t-1) x^{3}} d x,
$$

which proves that the sequence $\left\{r_{n+2}(2, t)\right\}_{n=0}^{\infty}$ is positive definite.

Remark. Note, that for $\eta(2,0)$ the cumulant sequence is $(2,1,0,0, \ldots)$, so the sequence $\left\{r_{n+2}(2,0)\right\}_{n=0}^{\infty}=(1,0,0, \ldots)$ is positive definite. Actually, $\eta(2,0)$, given by (12), is a translation of the Wigner semicircle distribution $\frac{1}{2 \pi} \sqrt{4-x^{2}} d x, x \in[-2,2]$. The free additive infinite divisibility of $\eta(2,0)$ was overlooked in [16], Corollary 7.1, where $\eta(2,0)$ was denoted $\mu(2,2)$.

Example 1. Define a sequence $a_{n}$ by $a_{0}:=1$ and $a_{n}:=3^{n} \cdot r_{n}(2,4 / 3)$ for $n \geq 1$ :

$$
1,2,5,16,64,304,1632,9552,59520,388720,2632864, \ldots
$$

Applying (32) for $t=4 / 3$ we obtain

$$
a_{n}=\int_{1}^{9} x^{n} \frac{\sqrt{(x-1)(9-x)^{3}}}{2 \pi x^{3}} d x .
$$

Its generating function is

$$
\sum_{n=0}^{\infty} a_{n} z^{n}=1+R_{\eta(2,4 / 3)}(3 z)=\frac{1+18 z-27 z^{2}+\sqrt{(1-z)(1-9 z)^{3}}}{2} .
$$

Example 2. Now let us consider the binomial transform of $a_{n}$ :

$$
b_{n}:=\sum_{k=0}^{n}(-1)^{n-k}\left(\begin{array}{l}
n \\
k
\end{array}\right) a_{k} .
$$

The corresponding density function is that of the sequence $a_{n}$ translated by -1 , so

$$
b_{n}=\int_{0}^{8} x^{n} \frac{\sqrt{x(8-x)^{3}}}{2 \pi(x+1)^{3}} d x
$$

For the generating function we have

$$
\sum_{n=0}^{\infty} b_{n} z^{n}=\sum_{k=0}^{\infty} a_{k}(-1)^{k} \sum_{n=k}^{\infty}\left(\begin{array}{l}
n \\
k
\end{array}\right)(-z)^{n}
$$




$$
=\sum_{k=0}^{\infty} a_{k} \frac{z^{k}}{(1+z)^{k+1}}=\frac{1}{1+z}\left(1+R_{\eta(2,4 / 3)}(3 z /(1+z))\right),
$$

so from 34

$$
\sum_{n=0}^{\infty} b_{n} z^{n}=\frac{1+20 z-8 z^{2}+\sqrt{(1-8 z)^{3}}}{2(1+z)^{3}} .
$$

This proves that $b_{n}$ coincides with $A 022558$ in OEIS:

$$
1,1,2,6,23,103,512,2740,15485,91245,555662, \ldots \text {, }
$$

which counts the permutations of length $n$ which avoid the pattern 1342, see Theorem 2 in [5].

\subsection{The case $p=3$.}

\section{Proposition 4.3.}

$$
R_{\eta(3, t)}(z)=\frac{z\left(4-7 t+4 t^{2}-2 z\right)-(t-1)^{2}+\left(1-2 t+t^{2}-t z\right) \sqrt{1-4 t z}}{2(t+z-1)^{2}}
$$

and the distribution $\eta(3, t)$ is infinitely divisible with respect to the additive free convolution if and only if $1 / 2 \leq t \leq 3 / 2$.

Proof. The proof is similar as for $p=2$. First we find $R_{\eta(3, t)}$ by solving the equation

$$
S_{\eta(3, t)}\left(R_{\eta(3, t)}(z)\right) R_{\eta(3, t)}(z)=z,
$$

with the condition that $R_{\eta(3, t)}(0)=0$. Then we find out that

$$
1+R_{\eta(3, t)}(z)=\frac{(t-1)^{2}+t z(4 t-3)+\left(1-2 t+t^{2}-t z\right) \sqrt{1-4 t z}}{2(t+z-1)^{2}}
$$

is the moment generating function for the density

$$
\frac{\left(t-x(t-1)^{2}\right) \sqrt{4 t-x}}{2 \pi(t x-x+1)^{2} \sqrt{x}}, \quad x \in[0,4 t]
$$

which is positive provided $1 / 2 \leq t \leq 3 / 2$.

Example. The sequence $a_{n}=A 220910(n)$ :

$$
1,1,3,14,83,570,4318,35068,299907,2668994,24513578, \ldots
$$

counts matchings avoiding the pattern 231, see [4] for details. Its generating function equals

$$
M(z)=\sum_{n=0}^{\infty} a_{n} z^{n}=\frac{1+36 z+\sqrt{(1-12 z)^{3}}}{2(1+4 z)^{2}}=1+R_{\eta(3,3 / 2)}(2 z),
$$

so we have $a_{n}=2^{n} \cdot r_{n}(3,3 / 2)$ for $n \geq 1$. Therefore these numbers can be represented as moments:

$$
a_{n}=\int_{0}^{12} x^{n} \frac{\sqrt{(12-x)^{3}}}{2 \pi(x+4)^{2} \sqrt{x}} d x .
$$

Now we are going to prove a recurrence relation, which was was conjectured by R. J. Mathar (see OEIS, entry A220910, Aug. 04 2013). 
Proposition 4.4. For $n \geq 2$ we have

$$
n a_{n}=(8 n-34) a_{n-1}+24(2 n-3) a_{n-2} .
$$

Proof. One can check that the generating function satisfies differential equation:

$$
\left(1-8 z-48 z^{2}\right) M^{\prime}(z)+(26-24 z) M(z)=27 .
$$

The coefficient at $z^{n-1}$ on the left hand side is equal to

$$
n a_{n}-8(n-1) a_{n-1}-48(n-2) a_{n-2}+26 a_{n-1}-24 a_{n-2}
$$

for $n \geq 2$, which gives 41).

Now we will provide two formulas for $a_{n}=A 220910(n)$.

Proposition 4.5.

$$
\begin{gathered}
a_{n}=\frac{1-8 n}{2}(-4)^{n}+\left(\begin{array}{c}
2 n \\
n
\end{array}\right) \sum_{k=0}^{n} \frac{3^{n+1}(k+1) \prod_{i=0}^{k-1}(n-i)}{8(-3)^{k} \prod_{i=0}^{k+1}(n-i-1 / 2)} \\
=\frac{(-4)^{n}(1-8 n)}{16}\left[8-\sum_{k=0}^{n+1} \frac{(-3)^{k}}{k !} \prod_{i=0}^{k-1}(i-3 / 2)\right]+\left(\begin{array}{c}
2 n \\
n
\end{array}\right) \frac{3^{n+3}}{32(n+1)} .
\end{gathered}
$$

Proof. Putting $x=12 t$ in 40 and applying formula (15.6.1) from [20] we get

$$
\begin{aligned}
a_{n} & =\frac{9 \cdot 12^{n}}{2 \pi} \int_{0}^{1} \frac{t^{n-1 / 2}(1-t)^{3 / 2}}{(1+3 t)^{2}} d t \\
& =\frac{27(2 n) ! 3^{n}}{8 n !(n+2) !}{ }_{2} F_{1}(2, n+1 / 2 ; n+3 \mid-3) .
\end{aligned}
$$

From (15.8.2) in [20] and from the identities

$$
\frac{\Gamma(n-3 / 2)}{\Gamma(n+1 / 2)}=\frac{4}{(2 n-3)(2 n-1)}, \quad \frac{\Gamma(3 / 2-n)}{\Gamma(5 / 2)}=\frac{(-2)^{n+1}(2 n-1)}{3(2 n-1) ! !},
$$

we have

$$
\begin{gathered}
{ }_{2} F_{1}(2, n+1 / 2 ; n+3 \mid-3)=\frac{4(n+2) !}{9 n !(2 n-1)(2 n-3)}{ }_{2} F_{1}(2,-n ; 5 / 2-n \mid-1 / 3) \\
+\frac{(-2)^{n+1}(n+2) !(2 n-1)}{3^{n+3 / 2}(2 n-1) ! !}{ }_{2} F_{1}(n+1 / 2,-3 / 2 ; n-1 / 2 \mid-1 / 3) .
\end{gathered}
$$

Since

$$
{ }_{2} F_{1}(2,-n ; 5 / 2-n \mid z)=\sum_{k=0}^{n}(k+1) z^{k} \prod_{i=0}^{k-1} \frac{n-i}{n-5 / 2-i}
$$

and

$$
{ }_{2} F_{1}(n+1 / 2,-3 / 2 ; n-1 / 2 \mid z)=\frac{(2 n-2 n z-2 z-1) \sqrt{1-z}}{2 n-1}
$$

(see formula (15.4.9) in [20]), we obtain

$$
\begin{gathered}
{ }_{2} F_{1}(2, n+1 / 2 ; n+3 \mid-3)=\frac{n !(n+2) !(8 n-1)(-4)^{n+1}}{(2 n) ! 3^{n+3}} \\
+\frac{4(n+1)(n+2)}{9(2 n-1)(2 n-3)} \sum_{k=0}^{n} \frac{k+1}{(-3)^{k}} \prod_{i=0}^{k-1} \frac{n-i}{n-5 / 2-i},
\end{gathered}
$$


which leads to 42 .

For the second formula we apply the identity

$$
{ }_{2} F_{1}(2, b ; c \mid z)(1-z)=(b z-z-c+2){ }_{2} F_{1}(1, b ; c \mid z)+c-1,
$$

see (15.5.11) in [20], to (45) and get

$$
{ }_{2} F_{1}(2, n+1 / 2 ; n+3 \mid-3)=\frac{1-8 n}{8}{ }_{2} F_{1}(1, n+1 / 2 ; n+3 \mid-3)+\frac{n+2}{4} .
$$

Applying formula (123), page 462, from [23]:

$$
{ }_{2} F_{1}(1, b ; m+1 \mid z)=\frac{m !}{z^{m}(b-1) \ldots(b-m)}\left((1-z)^{m-b}-\sum_{k=0}^{m-1} \frac{z^{k}}{k !} \prod_{i=0}^{k-1}(b+i-m)\right),
$$

with $b=n+1 / 2, m=n+2, z=-3$, and using the identity

$$
4^{n+1} n !(n+1 / 2-1) \ldots(n+1 / 2-n-2)=3(2 n) !
$$

we get 43 .

\section{REFERENCES}

[1] N. Alexeev, F. Götze and A. Tikhomirov, Asymptotic distribution of singular values of powers of random matrices, Lith. Math. J. 50 (2010), 121-132.

[2] D. Armstrong, Generalized Noncrossing Partitions and Combinatorics of Coxeter Groups, Memoirs of the American Mathematical Society, 202 (2009) 159 pp.

[3] T. Banica, S. Belinschi, M. Capitaine and B. Collins, Free Bessel laws, Canadian J. Math. 63 (2011), 3-37.

[4] Jonathan Bloom and Sergi Elizalde, Pattern avoidance in matchings and partitions, The Electronic Journal of Combinatorics 202 (2013) \#5.

[5] M. Bona, Exact enumeration of 1342-avoiding permutations; A close link with labeled trees and planar maps, J. Combinatorial Theory, A80 (1997), 257-272.

[6] M. Bousquet-Mélou and G. Schaeffer, Enumeration of planar constellations, Adv. in Appl. Math. 24 (2000), 337-368.

[7] P. H. Edelman, Chain enumeration and non-crossing partitions, Discrete Math. 31 (1980), 171180.

[8] P. J. Forrester and D.-Z. Liu, Raney distributions and random matrix theory, J. Stat. Phys. 158 (2015), 1051-1082.

[9] R. L. Graham, D. E. Knuth and O. Patashnik, Concrete Mathematics. A Foundation for Computer Science, Addison-Wesley, New York, 1994.

[10] U. Haagerup and S. Möller, The law of large numbers for the free multiplicative convolution, Springer Proceedings in Mathematics and Statistics, Vol. 58, ed. T. M. Carlsen et al., Springer, Berlin, 2014.

[11] D. Liu, C. Song and Z. Wang, On explicit probability densities associated with Fuss-Catalan numbers, Proc. Amer. Math. Soc. 139/10 (2011), 3735-3738.

[12] J.-G. Liu and R. Pego, On generating functions of Hausdorff moment sequences, to appear in Trans. Am. Math. Soc.

[13] W. Młotkowski, Fuss-Catalan numbers in noncommutative probability, Documenta Math. 15 (2010), 939-955.

[14] W. Młotkowski, K. A. Penson and K. Życzkowski, Densities of the Raney distributions, Documenta Mathematica 18 (2013), 1573-1596.

[15] W. Młotkowski and K. A. Penson, The probability measure corresponding to 2-plane trees, Probability nad Mathematical Statistics, 33/2 (2013), 255-264.

[16] W. Młotkowski and K. A. Penson, Probability distributions with binomial moments, Infinite Dimensional Analysis, Quantum Probability and Related Topics, 17/2 (2014) 1450014, 32pp.

[17] W. Młotkowski, M. A. Nowak, K. A. Penson and K. Życzkowski Spectral density of generalized Wishart matrices and free multiplicative convolution, Phys. Rev. E 92 (2015) 012121. 
[18] T. Neuschel, Plancherel-Rotach formulae for average characteristic polynomials of products of Ginibre random matrices and the Fuss-Catalan distribution, Random Matrices Theory Appl. 3 (2014), 1450003, 18 pp.

[19] A. Nica and R. Speicher, Lectures on the Combinatorics of Free Probability, Cambridge University Press, Cambridge, 2006.

[20] F. W. J. Olver, D. W. Lozier, R. F. Boisvert and C. W. Clark, NIST Handbook of Mathematical Functions, Cambridge University Press, Cambridge, 2010.

[21] K. A. Penson and A. I. Solomon, Coherent states from combinatorial sequences, pp. 527-530 in Quantum theory and symmetries, Kraków 2001, (World Sci. Publ., River Edge, NJ, 2002).

[22] K. A. Penson and K. Życzkowski, Product of Ginibre matrices: Fuss-Catalan and Raney distributions, Phys. Rev. E 83 (2011) 061118, 9 pp.

[23] A. P. Prudnikov, Yu. A. Brychkov and O. I. Marichev, Integrals and Series, Gordon and Breach, Amsterdam, 1998, Vol. 3: More special functions.

[24] J. H. Przytycki and A. S. Sikora, Polygon Dissections and Euler, Fuss, Kirkman, and Cayley Numbers, Journal of Combinatorial Theory, Series A 92, Issue 1, (2000) 68-76.

[25] T. Simon, Comparing Fréchet and positive stable laws, Electron. J. Probab. 19 (2014) no. 16, 1-25.

[26] N. J. A. Sloane, The On-Line Encyclopedia of Integer Sequences, (2015), published electronically at: http://oeis.org/.

[27] Alison Schuetz and Gwyneth Whieldon, Polygonal Dissections and Reversions of Series, arXiv:1401.7194.

[28] D. V. Voiculescu, K. J. Dykema, A. Nica, Free random variables, CRM, Montréal, 1992.

Instytut Matematyczny, Uniwersytet WrocŁaWski, Plac Grunwaldzki 2/4, 50-384 WrocŁaW, Poland

E-mail address: mlotkow@math.uni.wroc.pl

Sorbonne Universités, Université Pierre et Marie Curie, Laboratoire de Physique Théorique de la Matière Condensée (LPTMC), CNRS UMR 7600, Tour 13 - 5ième Ét., Bô̂te Courrier 121, 4 Place Jussieu, F 75252 Paris Cedex 05, France

E-mail address: penson@lptl.jussieu.fr 